\title{
Cyclophosphamide and low-dose prednisolone in idiopathic pulmonary fibrosis and fibrosing nonspecific interstitial
} pneumonia

\author{
Y. Kondoh*, H. Taniguchi*, T. Yokoi ${ }^{\#}$, O. Nishiyama*, T. Ohishi ${ }^{\mp}$, T. Kato ${ }^{+}$, K. Suzuki $^{\S}$ and R. Suzuki ${ }^{f}$
}

ABSTRACT: The present study compared the efficacy of cyclophosphamide combined with lowdose prednisolone in the treatment of idiopathic pulmonary fibrosis (IPF) with efficacy in idiopathic fibrosing nonspecific interstitial pneumonia fibrosing (NSIP).

A total of 27 patients with IPF and 12 patients with fibrosing NSIP were included in this study. All patients had undergone surgical lung biopsy. The diagnoses were made based on clinical, radiological and pathological findings. All patients were treated with intermittent pulse therapy with methylprednisolone for 4 weeks, followed by cyclophosphamide with low-dose prednisolone.

According to pulmonary function tests, four of 27 patients with IPF had improved, 22 remained unchanged, and one had worsened at the completion of pulse therapy. After 1 yr of combination therapy, four of 27 patients had improved, 14 remained unchanged, and nine had worsened. After pulse therapy, four of 12 patients with fibrosing NSIP had improved, and eight remained unchanged. After $1 \mathrm{yr}$ of combination therapy, eight of 12 patients had improved, four remained unchanged, and none had worsened. Median survival of IPF patients was 4.1 yrs, which is significantly worse than that of fibrosing NSIP patients.

In conclusion, patients with fibrosing nonspecific interstitial pneumonia had a more favourable response to combination therapy and a better survival than those with idiopathic pulmonary fibrosis.

KEYWORDS: Cyclophosphamide, fibrosing nonspecific interstitial pneumonia, idiopathic pulmonary fibrosis, immunosuppressant therapy, usual interstitial pneumonia

diopathic pulmonary fibrosis (IPF) is the most common type of interstitial lung disease

$[1,2]$. Generally, IPF is defined as a progressive, fibrosing inflammatory disease of unknown cause that involves the lung parenchyma. Whilst diagnosis is most often based on clinical and radiological findings without histological confirmation [3, 4], considerable advances have recently been made in characterising the underlying histological appearances of interstitial pneumonias [5-9].

Nonspecific interstitial pneumonia (NSIP) is characterised as a temporally uniform interstitial pneumonia, different from the heterogeneous appearance of usual interstitial pneumonia (UIP) [7]. Whilst NSIP was initially considered a "wastebasket" category for a variety of unclassifiable interstitial pneumonias [7], several studies indicated that patients with NSIP have a better outcome than those with UIP and that NSIP deserves to be considered as a clinicopathological entity $[10,11]$. After the definition of NSIP was established, the diagnostic category of UIP became more restricted and was redefined [12]. The entity UIP now has a much worse prognosis than NSIP or interstitial pneumonia with other histological patterns [8, 11, 13, 14]. The recent American Thoracic Society (ATS)/European Respiratory Society (ERS) consensus statement concluded that UIP is the histological pattern associated with IPF, and the histological pattern of NSIP identifies it as an entity that should be viewed as distinct from IPF, although it can present clinically in a fashion identical to IPF [15].

Corticosteroids were long considered the standard therapy for IPF, but therapeutic responses are usually partial and transient [16]. JOHNSON et al. [17] conducted a randomised, controlled trial comparing treatment with prednisolone alone with that of cyclophosphamide and

\section{AFFILIATIONS}

*Dept of Respiratory and Allergic Medicine, Tosei General Hospital, \#Dept of Respiratory and Allergic Medicine, Toyohashi Municipal Hospital, and

"Dept of Respiratory Medicine,

Toyota Medical Corporation, Kariya General Hospital, Aichi,

${ }^{+}$Dept of Medicine, Meijo Hospital, ${ }^{\text {s}}$ Dept of Laboratory Medicine, Nagoya University School of Medicine, and

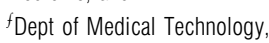
Nagoya University School of Medicine, Nagoya, Japan.

CORRESPONDENCE

Y. Kondoh

Dept of Respiratory and Allergic Medicine

Tosei General Hospital

160 Nishioiwake-cho

Seto

Aichi, 489-8642

Japan

Fax: 81561829139

E-mail: tosei-lung@deluxe.ocn.ne.jp

Received:

June 182004

Accepted after revision:

November 142004

European Respiratory Journal Print ISSN 0903-1936

Online ISSN 1399-3003 
low-dose prednisolone in combination, and the findings indicated a possible survival advantage for patients treated with cyclophosphamide and low-dose prednisolone. In a study by RAGHU et al. [18] on the survival of patients randomised to therapy, either with azathioprine plus low-dose prednisone or with prednisone alone, age-adjusted analysis indicated a significant survival advantage in patients receiving the combined regimen. Based on these findings, the combination of a corticosteroid with a cytotoxic agent is the therapy recommended in the ATS/ERS consensus statement concerning current diagnosis and treatment of IPF [15]. Although these studies demonstrate the benefits of therapy combining a corticosteroid with an immunosuppressant, the proportion of patients in these studies who had UIP, as currently defined, is unclear [12].

Idiopathic NSIP, especially with a fibrosing pattern, resembles IPF/UIP clinically, physiologically and radiologically. As a result, many patients with NSIP were previously diagnosed with IPF $[8,9,12,14]$. Although several initial reports indicated that NSIP carries a better prognosis than IPF/UIP $[7,8,11]$, more recent studies emphasised that cases with fibrotic NSIP (i.e. groups II and III) do not necessarily have a good outcome $[13,19]$. However, these studies did not assess therapeutic response and survival using a standardised therapeutic regimen.

In the present investigation, which compared the outcome in patients with histologically confirmed IPF/UIP and those with fibrosing NSIP, all patients had clinical manifestations compatible with IPF and received four courses of pulse steroid therapy, followed by cyclophosphamide combined with lowdose prednisolone, which is now the recommended therapy for IPF.

\section{METHODS}

\section{Patient selection}

The present authors prospectively treated patients with idiopathic intersitital pneumonia, confirmed by surgical lung biopsy (SLB), whose clinical features were consistent with IPF. Selection as a patient required the following: 1) exclusion of other known causes of interstitial lung disease, including drug toxicities, environmental exposures and collagen vascular diseases; 2) bibasilar reticular abnormalities on conventional chest radiographs or high-resolution computed tomography scans; and 3) abnormal pulmonary function studies showing restriction (reduced total lung capacity, or reduced vital capacity (VC) with a normal or increased forced expiratory volume in one second/forced VC ratio) and/or impaired gas exchange (increased alveolar-arterial pressure difference for oxygen, decreased arterial oxygen tension with rest or exercise, or decreased carbon monoxide diffusing capacity of the lung) $[15,19]$. All patients underwent SLB at Tosei General Hospital, Nagoya University Hospital, Toyohashi Municipal Hospital, Kariya General Hospital, Komaki Municipal Hospital or Handa Municipal Hospital, Aichi, Japan, between January 1991 and January 1998. They all received the same therapeutic regimen. In July 2003, all SLB specimens were reviewed by a pathologist according to the currently redefined criteria for idiopathic interstitial pneumonias [15, 19], and patients with UIP and fibrosing NSIP were included in the current study. Patients who had received any corticosteroids or immunosuppressant drugs prior to lung biopsy were ineligible. The number of eligible patients was 39 , including 27 with the histological pattern of UIP and 12 with that of fibrosing NSIP.

\section{Therapeutic regimen and evaluation}

After confirmatory lung biopsy, all patients included in the present study were treated for 4 weeks with intermittent highdose parenteral methylprednisolone $\left(1,000 \mathrm{mg} \cdot\right.$ day $^{-1}$ for 3 days at 1-week intervals, i.e. pulse therapy), followed by combination therapy for $1 \mathrm{yr}$ with cyclophosphamide (1$2 \mathrm{mg} \cdot \mathrm{kg} \cdot \mathrm{day}^{-1}$ ) plus low-dose prednisolone $(20 \mathrm{mg}$ on alternate days). Since large doses of corticosteroids are thought to be more effective than conventional doses, high-dose pulse corticosteroid therapy was used for the first phase of treatment in these patients. Short-term response to the 4 weeks of pulse therapy was assessed for usefulness in predicting the response to 1 yr of combination therapy.

For maintenance therapy, the current authors treated patients according to the method of JOHNSON et al. [17] which is now the therapy recommended by the ATS/ERS consensus statement for IPF [15].

Pulmonary function testing just prior to treatment (within 1 month), at 1 month of treatment immediately following 4 weeks of high-dose parenteral methylprednisolone therapy, and after $1 \mathrm{yr}$ of combination therapy was used to assess the therapeutic response. Improvement in pulmonary function was defined as $\geqslant 10 \%$ increase in the percentage of predicted VC (VC \% pred) achieved. A decrease of similar magnitude was considered as deterioration. Patients who did not demonstrate improvement or deterioration were considered unchanged.

\section{Outcome}

Survival status in July 2003 was established from clinical records.

\section{Statistical methods}

Data are presented as mean \pm SD or as median (interquartile range). Differences between subjects in the two disease-defined groups were examined by the Mann-Whitney U-test or the Chi-squared test. One-way repeated-measures ANOVA was used to evaluate changes in VC \% pred. Kaplan-Meier analysis was used to compare survival between the groups.

\section{RESULTS}

\section{Clinical and laboratory features}

The IPF/UIP group included 20 males and seven females with a mean age of 56 yrs. The NSIP group included four males and eight females with a mean age of 55.3 yrs. Clinical features of both groups are summarised in table 1 . Significant differences were evident between the two groups in sex and duration of symptoms before biopsy ( $p=0.031$ and $p=0.004$, respectively).

Bronchoalveolar lavage (BAL) was performed upon first presentation in all patients. There were significant differences in numbers of macrophages, neutrophils, lymphocytes, eosinophils and OKT4/OKT8 (helper cell/suppressor cell, respectively) between the two groups upon analysis of the lavage fluid (table 2). 


\begin{tabular}{lccc}
\hline TABLE 1 & $\begin{array}{l}\text { Demographic characteristics, clinical features, } \\
\text { laboratory findings, and pulmonary function test } \\
\text { results }\end{array}$ \\
& IPF/UIP & Fibrosing NSIP & p-value \\
\hline Subjects n & 27 & 12 & \\
Age yr & $56.0 \pm 10.9$ & $55.3 \pm 6.6$ & NS \\
Male & $20(74)$ & $4(33)$ & 0.031 \\
Rales present & $24(89)$ & $12(100)$ & NS \\
Digital clubbing present & $10(37)$ & $1(8)$ & 0.122 \\
Duration of symptom months & $25.5 \pm 25.3$ & $5.5 \pm 5.4$ & 0.004 \\
Smoker & $20(74)$ & $4(33)$ & NS \\
White blood cells $\cdot \mu$ L & $7204 \pm 2148$ & $7675 \pm 790$ & NS \\
LDH elevation & $10(37)$ & $6(50)$ & NS \\
ANA positive & $5(19)$ & $3(25)$ & NS \\
RF positive & $4(15)$ & $4(33)$ & NS \\
VC \% pred & $66.2 \pm 18.2$ & $56.7 \pm 12.7$ & 0.162 \\
DL,Co \% pred & $60.8 \pm 18.2$ & $68.7 \pm 21.9$ & 0.199 \\
Pa, O $_{2}$ mmHg & $82.1 \pm 14.2$ & $77.4 \pm 12.6$ & 0.181 \\
\hline
\end{tabular}

Data are presented as $\mathrm{n}(\%)$ or mean $\pm \mathrm{SD}$, unless otherwise stated. IPF idiopathic pulmonary fibrosis; UIP: usual interstitial pneumonia; NSIP: nonspecific interstitial pneumonia; LDH: lactic dehydrogenase; ANA: antinuclear antibody; RF:rheumatoid factor; VC: vital capacity; \% pred: per cent predicted; $\mathrm{DL}, \mathrm{CO}$ : carbon monoxide diffusing capacity of the lung; $\mathrm{Pa}_{\mathrm{a}} \mathrm{O}_{2}$ : arterial oxygen tension. p-values were determined by the Mann-Whitney U-test or the Chi-squared test. NS: nonsignificant.

\begin{tabular}{lccc} 
TABLE 2 & $\begin{array}{l}\text { Bronchoalveolar lavage fluid findings in patients } \\
\text { with idiopathic pulmonary fibrosis (IPF)/Usual } \\
\text { interstitial pneumonia (UIP) and those with } \\
\text { fibrosing nonspecific interstitial pneumonia (NSIP) }\end{array}$ \\
& IPF/UIP & Fibrosing NSIP & p-value \\
\hline & & & \\
Subjects $\mathbf{n}$ & 27 & 12 & \\
Cell counts $\times \mathbf{1 0}^{\mathbf{4}} \cdot \mathbf{m L}^{-\mathbf{1}}$ & $24.8(1.6-70.0)$ & $21.3(3.6-70.0)$ & NS \\
Macrophages \% & $92.0(50.0-98.3)$ & $64.4(11.0-90.3)$ & 0.001 \\
Neutrophils \% & $0.7(0.0-21.7)$ & $2.9(1.0-8)$ & 0.029 \\
Lymphocytes \% & $5.4(0.3-48.1)$ & $20.5(3.0-75.9)$ & 0.018 \\
Eosinophils \% & $0.3(0.0-5.8)$ & $3.0(0.0-13.7)$ & 0.018 \\
OKT4/8 \% & $1.86(0.10-11.56)$ & $0.51(0.10-4.86)$ & 0.015 \\
\hline
\end{tabular}

Data are presented as median (interquartile range), unless otherwise stated. OKT4/OKT8: helper cell/suppressor cell, respectively. NS: nonsignificant.

\section{Effect of treatment}

In the group of patients with IPF/UIP, VC \% pred improved significantly from pre-treatment values after pulse therapy $(70.8 \pm 15.5 \%$ versus $74.9 \pm 16.2 \%, \mathrm{p}<0.005)$. However, this was not true when VC \% pred was compared between pre-treatment baseline data and data after $1 \mathrm{yr}$ of combination therapy $(70.8 \pm 15.5 \%$ versus $72.1 \pm 17.3 \%, p=0.52$; fig. 1$)$. In the group of patients with fibrosing NSIP, VC \% pred improved significantly after pulse therapy $(56.7 \pm 12.7 \%$ versus $64.2 \pm$ $15.7 \%, \mathrm{p}<0.01)$ and $1 \mathrm{yr}$ of combination therapy $(56.7 \pm 12.7 \%$ versus $77.2 \pm 16.1 \%, \mathrm{p}<0.001$; fig. 1 ).

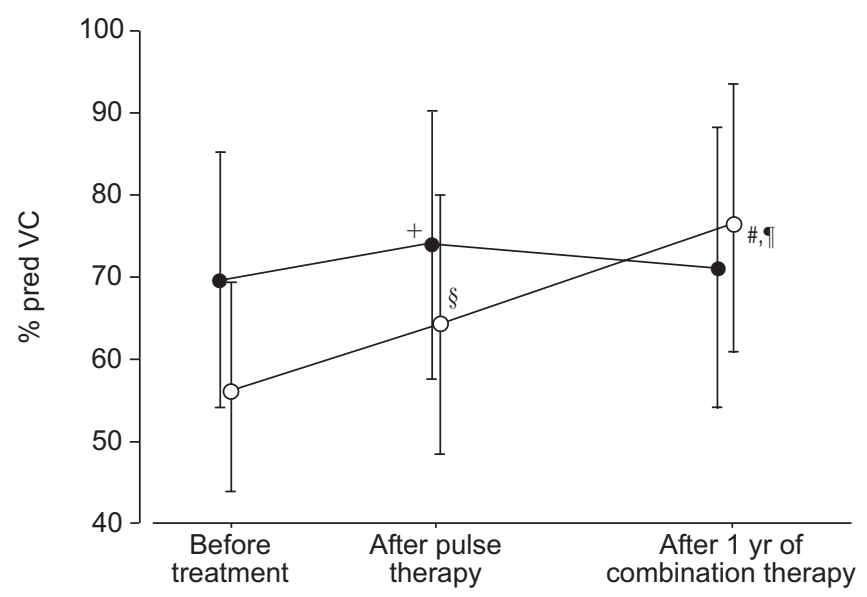

FIGURE 1. Changes in mean vital capacity (VC) $\pm S D$. One-way repeatedmeasures ANOVA revealed a significant difference between idiopathic pulmonary fibrosis (IPF)/usual interstitial pneumonia (UIP; - ) and fibrosing nonspecific interstitial pneumonia (NSIP; $\bigcirc$ ). In the group of patients with IPF/UIP, there was a significant difference in VC \% pred between pre-treatment values and those after pulse therapy. In the group of patients with NSIP, there was a significant difference in VC \% pred between pre-treatment value and after pulse therapy, pre-treatment value and $1 \mathrm{yr}$ of combination therapy, and after pulse therapy and $1 \mathrm{yr}$ of combination therapy. ${ }^{+}: p<0.005$ versus IPF/UIP before treatment; ${ }^{s}: p<0.01$ versus NSIP before treatment; \#: $p<0.001$ versus NSIP before treatment; $\bullet: p<0.005$ versus NSIP after pulse therapy.

Numbers of patients responding to pulse therapy and subsequent combination therapy for $1 \mathrm{yr}$ are shown in table 3. Improvement was evident at completion of pulse therapy in four of 27 patients with IPF/UIP (15\%). Of 27 patients, $22(81 \%)$ remained unchanged, and one of $27(4 \%)$ worsened. After $1 \mathrm{yr}$ of combination therapy, four of $27(15 \%)$ had improved, 14 of $27(52 \%)$ remained unchanged, and nine of $27(33 \%)$ had worsened. Three patients who died during the yr-long combination therapy were included among the nine patients who worsened.

After pulse therapy, four of $12(33 \%)$ patients with fibrosing NSIP had improved, eight of $12(67 \%)$ remained unchanged, and no patients had worsened. After $1 \mathrm{yr}$ of combination therapy, eight of $12(67 \%)$ patients had improved, four of 12 $(33 \%)$ remained unchanged, and none had worsened.

No patients with IPF/UIP showed improvement on the chest radiograph, whilst improvement was observed in all patients with fibrosing NSIP who showed improvement in VC \% pred.

\section{Long-term outcome: response to therapy}

Survival and respiratory status in July 2003 was ascertained for all patients in each group. After $1 \mathrm{yr}$ of cyclophosphamide with low-dose predonisolone therapy, cyclophosphamide was gradually tapered off, based on the clinical response and tolerance (mean duration 21 months; range 3.5-60), due to concern over side-effects, especially secondary malignancy.

Among patients with IPF/UIP, none remained improved, seven of $27(26 \%)$ remained unchanged, and 20 of $27(74 \%)$ had worsened including 18 (67\%) who had died (mean follow-up 


\begin{tabular}{|c|c|c|c|c|c|c|c|}
\hline \multirow[t]{3}{*}{ TABLE 3} & \multicolumn{7}{|c|}{$\begin{array}{l}\text { Response upon completing } 1 \text { yr of combined therapy in patients with idiopathic pulmonary fibrosis (IPF)/usual interstitial } \\
\text { pneumonia (UIP) and those with fibrosing nonspecific interstitial pneumonia (NSIP), according to initial pulse therapy } \\
\text { response }\end{array}$} \\
\hline & & \multicolumn{3}{|c|}{ IPF/UIP 1 yr } & \multicolumn{3}{|c|}{ Fibrosing NSIP 1 yr } \\
\hline & & Improved & Unchanged & Worsened & Improved & Unchanged & Worsened \\
\hline \multicolumn{2}{|c|}{ Improved after pulse therapy } & 1 & 2 & 1 & 4 & 0 & 0 \\
\hline \multicolumn{2}{|c|}{ Unchanged after pulse therapy } & 3 & 12 & 7 & 4 & 4 & 0 \\
\hline \multicolumn{2}{|c|}{ Worse after pulse therapy } & 0 & 0 & 1 & 0 & 0 & 0 \\
\hline \multicolumn{2}{|l|}{ Total } & 4 & 14 & 9 & 8 & 4 & 0 \\
\hline
\end{tabular}

duration, 49.5 months; range 3.5-113). A total of 12 patients with IPF died as a result of advancing disease (chronic respiratory failure), and six patients died from acute respiratory failure of unknown cause, or so-called acute exacerbation of IPF [20, 21]. Among patients with NSIP, five of $12(42 \%)$ remained improved, four of $12(33 \%)$ remained unchanged, and three of $12(25 \%)$ had worsened (mean follow-up duration, 91.8 months; range 60-148). Among the three patients who worsened, one patient, who was unchanged both after 4 weeks pulse therapy and $1 \mathrm{yr}$ of combination therapy, died as a result of advancing disease after having overcome six occurrences of acute respiratory failure of unknown cause. The other two patients, who had improved after $1 \mathrm{yr}$ of combination therapy, worsened 1.5 and 2 yrs after completion of 2 and 3 yrs of combination therapy, respectively. They showed little response to re-administration of combination therapy. Kaplan-Meier analysis showed a significant difference in survival between groups (Logrank test, $\mathrm{p}=0.002$; fig. 2).

\section{Toxicity}

No severe adverse reactions were observed during steroid pulse therapy. A total of eight patients $(21 \%)$ had adverse effects attributable to cyclophosphamide. These included: one

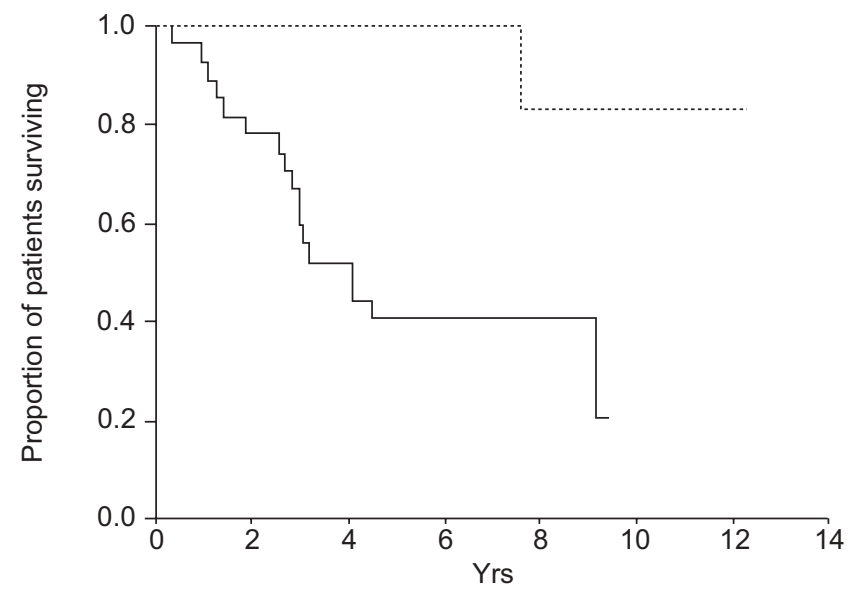

FIGURE 2. Kaplan-Meier survival curves of patients with idiopathic pulmonary fibrosis/usual interstitial pneumonia $(-)$ and fibrosing nonspecific interstitial pneumonia/fibrosis $(\cdots \cdots)$. with haemorrhagic cystitis, two with leukopenia, one with myelodysplastic syndrome, two with herpes zoster, one with alopecia, and one with aspergilloma. In all, discontinuation of therapy was required in five patients $(13 \%)$. In two patients with haematologic toxicity, the reduced neutrophil counts returned to normal once the dose was reduced. One IPF patient who suffered myelodysplastic syndrome died because of an opportunistic infection.

\section{DISCUSSION}

The present investigation compared the differences in response to a uniform treatment regimen with steroid pulse therapy followed by combined cyclophosphamide and low-dose predonisolone therapy, as well as differences in survival, between patients with IPF/UIP and fibrosing NSIP. Since the clinical manifestations of fibrosing NSIP in the current study mimicked IPF $[5,8,12,14]$, the presented NSIP patients might have been classified as IPF without performing lung biopsy. In the present study, patients with IPF/UIP showed small but significant improvement after 4 weeks of pulse therapy, but improvement was not maintained by combination therapy for 1 yr. In contrast, patients with fibrosing NSIP showed a better therapeutic response compared with patients with IPF/UIP, both after 4 weeks of pulse therapy and after completing $1 \mathrm{yr}$ of combination therapy. These results indicate that, in IPF/ UIP, treatment by pulse and combination therapy has limited effect, whilst in fibrosing NSIP combination maintenance therapy can usually produce improvement.

The proportions of patients showing improvement, stability, and deterioration in response to pulse therapy were not significantly different between patients with IPF/UIP and those with fibrosing NSIP, so response to pulse therapy had no differential diagnostic significance. After 1 yr of combination therapy, three of four IPF/UIP patients who had initially improved after 4 weeks of pulse therapy reverted to an unchanged status, and two of 14 initially unchanged patients improved. In IPF, responses to initial pulse therapy did not correlate with response after $1 \mathrm{yr}$ of combination therapy. In contrast, eight of 12 patients with fibrosing NSIP who did not show any worsening during pulse therapy (four of four with improvement, four of eight with no change) had improved after $1 \mathrm{yr}$ of therapy. Therefore, the current authors surmise that improvement at 4 weeks predicts improvement at $1 \mathrm{yr}$ for fibrosing NSIP patients. 
In many previous studies of IPF, $10-30 \%$ of patients showed initial objective improvement with corticosteroid therapy [22, 23]. However, the data are difficult to interpret as a SLB was not always obtained. Although JoHNSON et al. [17] reported beneficial effects of cyclophosphamide and prednisolone, several interstitial pneumonias other than IPF/UIP are suspected to have been included in that study. The results of the present study showed that eight of 12 of fibrosing NSIP cases $(67 \%)$, but only four of 27 of IPF/UIP cases $(15 \%)$, responded to $1 \mathrm{yr}$ of therapy. Additional differences between IPF/UIP and fibrosing NSIP were observed in patients with improvement. In IPF/UIP patients, chest radiograph findings did not improve even in functionally improved patients. Furthermore, almost all IPF/UIP patients, even all four cases with improvement, eventually showed a relentlessly progressive course. Thus, the presented results support recent suggestions that patients with marked improvement in previous IPF studies may have had a disorder other than IPF/UIP as it is currently defined [12]. The finding of progression in many patients with IPF/UIP, even after improvement, suggests limited and unsatisfactory effectiveness of current combination therapy for the disease.

The need for immunosuppressants in the treatment of NSIP is controversial. Although most patients reported by KATZENSTEIN and FIORELLi [7] had a good outcome, with most showing improvement after treatment with corticosteroids, some patients with NSIP were reported to receive immunosuppressive agents as the initial response to corticosteroids alone was insufficient. In a recent report by TRAVIS et al. [13], patients with idiopathic fibrosing NSIP had a poor long-term outcome (5-yr survival rate of $90 \%$ and a $10-\mathrm{yr}$ rate of $35 \%$ ). NicHOLSON et al. [24] also reported that in patients with clinically typical IPF, a histological pattern of fibrotic NSIP needs to be interpreted with caution, and does not assure a good outcome. Moreover, in patients with severe functional impairment, no survival difference was found between IPF and fibrotic NSIP [25]. Overall, the current authors suppose that patients with fibrosing NSIP should be treated initially by a combination of corticosteroid and immunosuppressant therapy to prevent development of an irreversible fibrosis. In the present study, early introduction of cyclophosphamide combined with low-dose prednisolone therapy for fibrosing NSIP resulted in a favourable outcome, so it appears to be an acceptable treatment for fibrosing NSIP. Further studies are needed to compare the effect of corticosteroid therapy with corticosteroid and immunosuppressant combination therapy for idiopathic fibrosing NSIP.

Upon analysis of BAL fluid, significant differences in the populations of macrophages, neutrophils and lymphocytes were also found between the two groups in the present study. NAGAI et al. [11] found more lymphocytes in BAL fluid from NSIP than UIP patients, whilst DANIIL et al. [14] reported that BAL fluid showed similar cell patterns in UIP and NSIP patients. Several previous reports have suggested that patients with IPF who had lymphocytosis in BAL fluid were more likely to respond to therapy $[26,27]$. Whether or not BAL fluid findings are related to therapeutic response and survival in patients with fibrosing NSIP is a question that requires further study.
In conclusion, patients with idiopathic pulmonary fibrosis/ usual interstitial pneumonia and those with fibrosing nonspecific interstitial pneumonia have similar clinical manifestations, but show significant differences in the response to combined prednisolone/cyclophosphamide therapy and survival. In idiopathic pulmonary fibrosis, combination of corticosteroid and immunosuppressive therapy has only limited efficacy. In contrast, the favourable therapeutic responsiveness and survival in the authors' fibrosing nonspecific interstitial pneumonia patients may suggest the efficacy of immunosuppressive therapy for fibrosing nonspecific interstitial pneumonia. Further studies are needed to determine the role of immunosuppressive therapy and longterm prognosis in fibrosing nonspecific interstitial pneumonia.

\section{ACKNOWLEDGEMENTS}

The authors would like to express their appreciation to $S$. Matsumoto, Y. Noda, T. Mori and N. Araki who cared for the study patients. The authors would also like to acknowledge the contributions of K. Matsuo for statistical support.

\section{REFERENCES}

1 Crystal RG. Idiopathic pulmonary fibrosis; clinical, histologic, radiographic, scintigraphic, cytologic and biochemical aspects. Ann Intern Med 1976; 85: 769-788.

2 Turner-Warwick M, Burrows BE, Johnson A. Cryptogenic fibrosing alveolitis: response to corticosteroid treatment and its effect on survival. Thorax 1980; 35: 593-599.

3 Ciultas DB, Zumwalt RE, Black WC, Sobonya RE. The epidemiology of interstitial lung diseases. Am J Respir Crit Care Med 1994; 150: 967-972.

4 Johnston IDA, Prescott RJ, Chalmers JC, Rudd RM. British Thoracic Society study of cryptogenic fibrosing alveolitis: current presentation and initial management. Thorax 1997; 52: $38-44$.

5 Epler GR, Coby TV, McLoud TC, Carrington C, Gaensler EA. Bronchiolotis obliterans organizing pneumonia. N Engl J Med 1985; 312: 152-158.

6 Katzenstein ALA, Myers JL, Mazur MT. Acute interstitial pneumonia: a clinicopathologic, ultrastructural, and cell kinetic study. Am J Surg Pathol 1986; 10: 256-267.

7 Katzenstein ALA, Fiorelli LF. Nonspecific interstitial pneumonia/fibrosis: histologic features and clinical significance. Am J Surg Pathol 1994; 18: 136-147.

8 Bjoraker JA, Ryu JH, Edwin MK, et al. Prognostic significance of histopathological subsets in idiopathic pulmonary fibrosis. Am J Respir Crit Care Med 1998; 157: 199-203.

9 Katzenstein ALA, Myers JL. Idiopathic pulmonary fibrosis: clinical relevance and pathologic classification. Am J Respir Crit Care Med 1998; 157: 1301-1315.

10 Cottin V, Donsbeck A, Revel D, Loire R, Cordier JF. Nonspecific interstitial pneumonia. Individualization of clinicopathologic entity in a series of 12 patients. Am J Respir Crit Care Med 1998; 158: 1286-1293.

11 Nagai S, Kitaichi M, Itoh H, Nishimura K, Izumi $T$, Colby TV. Idiopathic nonspecific interstitial pneumonia/ fibrosis: comparison with idiopathic pulmonary fibrosis and BOOP. Eur Respir J 1998; 12: 1010-1019. 
12 Ryu JH, Colby TV, Hartman TE. Idiopathic pulmonary fibrosis: current concepts. Mayo Clin Proc 1998; 73: 1085-1101.

13 Travis WD, Matsui K, Moss J, Ferrans V. Idiopathic nonspecific interstitial pneumonia: prognostic significance of cellular and fibrosing patterns. Am J Surg Pathol 2000; 24: 19-33.

14 Daniil ZD, Gilchrist FC, Nicholson AG, et al. A histologic pattern of nonspecific interstitial pneumonia is associated with a better prognosis than usual interstitial pneumonia in patients with cryptogenic fibrosing alveolitis. Am J Respir Crit Care Med 1999; 160: 899-905.

15 American Thoracic Society. Idiopathic pulmonary fibrosis: Diagnosis and treatment. International consensus statement. Am J Respir Crit Care Med 2000; 161: 646-664.

16 Mapel DW, Samet JM, Coultos DB. Corticosteroids and the treatment of idiopathic pulmonary fibrosis: past, present, and future. Chest 1996; 110: 1058-1067.

17 Johnson MA, Kwan S, Snell NJ, Nunn AJ, Darbyshire JH, Turner-Warwick M. Randomized controlled trial comparing prednisolone alone with cyclophosphamide and low dose prednisolone in combination in cryptogenic fibrosing alveolitis. Thorax 1989; 44: 280-288.

18 Raghu GW, Depaso WJ, Cain K, et al. Azathioprine combined with prednisone in the treatment of idiopathic pulmonary fibrosis: a prospective, double-blind randomized, placebo-controlled clinical trial. Am Rev Respir Dis 1991; 144: 291-296.

19 American Thoracic Society, European Respiratory Society. American Thoracic Society/European Respiratory Society International Multidisciplinary Consensus Classification of the Idiopathic Interstitial Pneumonias. Am J Respir Crit Care Med 2002; 165: 277-304.
20 Kondoh Y, Taniguchi H, Kawabata Y, Yokoi T, Suzuki K, Takagi K. Acute exacerbation in idiopathic pulmonary fibrosis. Analysis of clinical and pathologic findings in three cases. Chest 1993; 103: 1808-1812.

21 Ambrosini V, Cancellieri A, Chilosi M, et al. Acute exacerbation of idiopathic pulmonary fibrosis: report of a series. Eur Respir J 2003; 22: 21-26.

22 Carrington CB, Gaensler EA, Coutu RE, Fitzgerald MX, Gupter RG. Natural history and treated course of usual and desquamative interstitial pneumonia. New Engl J Med 1978; 298: 801-809.

23 Turner-Warwick M, Burrows BE, Johnson A. Cryptogenic fibrosing alveolitis: response to corticosteroid treatment and its effect on survival. Thorax 1980; 35: 593-599.

24 Nicholson AG, Colby TV, du Bois RM, Hansell DM, Wells AU. The prognostic significance of the histologic pattern of interstitial pneumonia in patients presenting with the clinical entity of cryptogenic fibrosing alveolitis. Am J Respir Crit Care Med 2000; 162: 2213-2217.

25 Latsi PI, du Bois RM, Nicholson AG, et al. Fibrotic idiopathic interstitial pneumonia: the prognostic value of longitudinal functional trends. Am J Respir Crit Care Med 2003; 168: 531-537.

26 Turner-Warwick M, Haslam PL. The value of serial bronchoalveolar lavages in assessing the clinical prognosis of patients with cryptogenic fibrosing alveolitis. Am Rev Respir Dis 1987; 135: 26-34.

27 Watters LC, Shwartz MI, Cherniack RM, et al. Idiopathic pulmonary fibrosis: pretreatment bronchoalveolar lavage cellular constituents and their relationships with lung histopathology and clinical response to therapy. Am Rev Respir Dis 1987; 135: 696-704. 